\title{
Extraction and Analysis of Document Examiner Features from Vector Skeletons of Grapheme 'th'
}

\author{
Vladimir Pervouchine and Graham Leedham \\ Forensics and Security Lab, \\ Nanyang Technological University, School of Computer Engineering, \\ N4-2C-77 Nanyang Avenue 639798, Singapore \\ \{pervouchine, asgleedham\}@ntu.edu.sg
}

\begin{abstract}
This paper presents a study of 25 structural features extracted from samples of grapheme 'th' that correspond to features commonly used by forensic document examiners. Most of the features are extracted using vector skeletons produced by a specially developed skeletonisation algorithm. The methods of feature extraction are presented along with the results. Analysis of the usefulness of the features was conducted and three categories of features were identified: indispensable, partially relevant and irrelevant for determining the authorship of genuine unconstrained handwriting. The division was performed based on searching the optimal feature sets using the wrapper method. A constructive neural network was used as a classifier and a genetic algorithm was used to search for optimal feature sets. It is shown that structural micro features similar to those used in forensic document analysis do possess discriminative power. The results are also compared to those obtained in our preceding study, and it is shown that use of the vector skeletonisation allows both extraction of more structural features and improvement the feature extraction accuracy from $87 \%$ to $94 \%$.
\end{abstract}

\section{Introduction}

Handwriting is a personal biometric that has long been considered to be unique to a person. Provided this statement is true, handwriting can be used to identify a person. The methods used by forensic document examiners to determine whether handwriting is genuine, forged, or disguised, are based on a set of established and well documented techniques [1-3]. The techniques have been derived from experience and are generally accepted by the various forensic laboratories. Forensic document analysis of handwriting is the examination of the design, shape, and structures of handwriting. Professional document examiners seek characteristics of handwriting that are consistent in a person's normal writing. These characteristics are called features [4, 3, 1 .

Whilst the techniques used by forensic document examiners are intuitively reasonable, they still lack a scientific basis. In recent court cases the scientific acceptability of forensic document examination that deals with handwriting analysis has been successfully challenged $[\underline{5}$. 
There is a need to determine whether it is possible to establish a sound scientific basis for forensic document analysis. To do this it is necessary to determine to what extent handwriting is unique to an individual and whether the methods of handwriting analysis practised by forensic experts can lead to correct and repeatable results. Since methods of forensic document examiners are based on structural features of handwriting, it is useful to investigate whether those features can be used for writer discrimination. This paper reports the experiments on automatic extraction of some of the document examiner features from images of handwritten grapheme 'th' followed by analysis of discriminating power of the features.

\section{Background}

\subsection{Performance of Forensic Document Examiners}

A number of tests have been performed under various conditions to demonstrate that the accuracy of writer identification by forensic document examiners is significantly higher than that of lay people [6, 7, 8, 9]. Establishing these facts means that techniques used by forensic document examiners may indeed allow them to identify authorship with observable accuracy. Hence it is necessary to verify the claim that it is the techniques and not something else (e.g. pure experience from having seen a lot of samples) that mainly contribute to the high writer identification accuracy demonstrated by the experts.

\subsection{Establishing Handwriting Individuality}

The problem of establishing the individuality of handwriting has received extensive study during recent years [10-12]. The individuality of handwriting is formally defined as "given two well-selected samples of handwriting, we can tell whether they were written by the same person or by two different people with a high degree of confidence." [13, 10. Further research has been carried out to study the discriminatory power of certain handwriting elements, particularly words [14, characters [15, and digits [16]. It has been shown that certain characters, especially those with ascenders and descenders, as well as capital letters bear more individual information than others which agrees with the statements of forensic document examiners [17,3]. It has been demonstrated that handwriting can be used to identify a person with high accuracy.

\subsection{Document Examiner and Computational Features}

The features of handwriting that are commonly used by forensic document examiners to determine the authorship are called document examiner features. Huber and Headrick summarised them in the list of 21 discriminating elements of handwriting 17. Many document examiner features are defined vaguely and thus do not allow reliable measurement with repeatable results.

Features of handwriting that are defined unambiguously and can be measured from handwriting images are called computational features. It is not necessary 
that a computational feature corresponds to any document examiner feature. For the study of handwriting individuality any computational features are suitable. However, the open question remains whether the methods used by forensic document examiners allow them to determine the authorship. To answer that question it is necessary to study how useful the document examiner features are for writer discrimination. For this purpose we formalise document examiner features, that is, map them into a set of computational features so that each computational feature represents some structural element of handwriting.

\section{$3 \quad$ Extraction of Features}

According to the classification of features of handwriting in relation to the scale of extraction all features can be divided into two categories: macro features and micro features [13. The macro features are those extracted from the whole document image, from lines, and from words up to the character level. Micro features are those extracted from short consistent character combinations (graphemes), characters, their sub-parts as well as between-character parts (ligatures). Micro features are thought to be more endowed with individual traits and are thought to be harder for the writers to change when attempting to forger or disguise their handwriting. This study is focused on micro features that correspond to document examiner features and which are formalised so that they can be extracted and measured unambiguously and accurately from handwriting images.

\subsection{Choice of Features}

Grapheme 'th' was chosen for the study because it is the most frequent grapheme in the English language [18. It accounts for around $4 \%$ of all two-character combinations. Also, our previous experiments showed that the discriminative power of the grapheme is higher than that of single characters $[19,20$. The features for study were selected so that they correspond to the structural micro features commonly used by forensic document examiners [17, 3, 1. Some of the features are shown in Figure 1, and the complete list of features is presented in Table1.

The feature set consists of geometrical characteristics of characters, various angular measures, loop characteristics, and stroke features. Definition of height and width as well as the relative position of the top point of ' $t$ ' $T$ and top point of 'h' $H$ are given in Figure 1, Position of t-bar was a binary feature and was equal to 1 for a t-bar crossing the stem and 0 in the cases of touching, detached, or absent t-bar. It was observed that writers who tend to produce t-bars touching the stem in grapheme 'th' also tend to produce disconnected t-bars in that same grapheme and vice versa. Slant of characters ' $t$ ' and ' $h$ ' was defined as the slant of their stems. Pseudo-pressure was calculated as the gray level of pixels. Retraced strokes were considered as loops hence the total number of loops and retraced strokes was measured. Straightness of strokes (t-bar, t-stem, and h-stem) was defined as the ratio of the total length of the stroke to the distance between its end points. Presence of loops at the top points of ' $t$ ' and ' $h$ ' stems was a binary feature having the value of 1 when a loop was present and the value of 0 


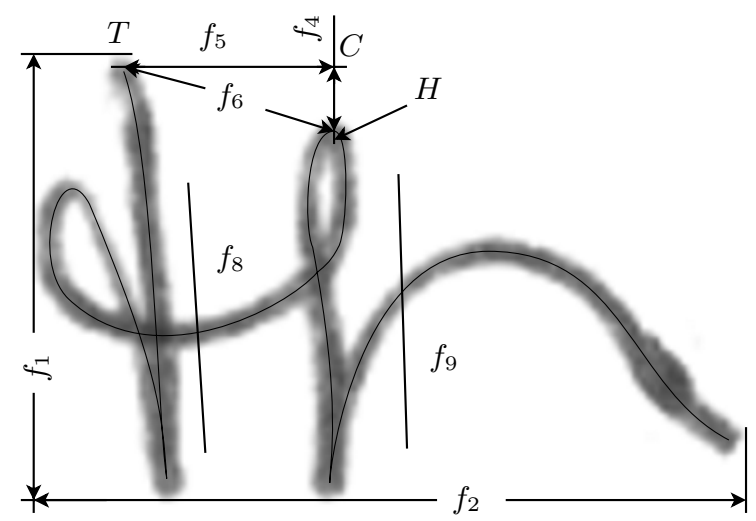

Fig. 1. Some features extracted from grapheme 'th'

Table 1. List of features extracted from grapheme 'th'

\begin{tabular}{||l|c||}
\hline Height & $f_{1}$ \\
\hline Width & $f_{2}$ \\
\hline Height to width ratio & $f_{3}$ \\
\hline Distance $H C$ & $f_{4}$ \\
\hline Distance $T C$ & $f_{5}$ \\
\hline Distance $T H$ & $f_{6}$ \\
\hline Angle between $T H$ and $T C$ & $f_{7}$ \\
\hline Slant of t & $f_{8}$ \\
\hline Slant of h & $f_{9}$ \\
\hline Position of t-bar & $f_{10}$ \\
\hline Connected / disconnected t and h & $f_{11}$ \\
\hline Average stroke width & $f_{12}$ \\
\hline Average pseudo-pressure & $f_{13}$ \\
\hline
\end{tabular}

\begin{tabular}{||l|l||}
\hline Standard deviation of pseudo-pressure & $f_{14}$ \\
\hline Standard deviation of stroke width & $f_{15}$ \\
\hline Number of strokes & $f_{16}$ \\
\hline Number of loops and retraced strokes & $f_{17}$ \\
\hline Straightness of t-stem & $f_{18}$ \\
\hline Straightness of t-bar & $f_{19}$ \\
\hline Straightness of h-stem & $f_{20}$ \\
\hline Presence of loop at top of t-stem & $f_{21}$ \\
\hline Presence of loop at top of h-stem & $f_{22}$ \\
\hline Maximum curvature of h-knee & $f_{23}$ \\
\hline Average curvature of h-knee & $f_{24}$ \\
\hline Relative size (diameter) of h-knee & $f_{25}$ \\
\hline
\end{tabular}

otherwise. Relative size (diameter) of h-knee was defined as the largest horizontal distance between the stem and knee strokes.

\subsection{Measurement of Feature Values}

The origin of the coordinate system is in the upper-left corner of an image, the abscissa $(x)$ axis is horizontal and directed to the right, the ordinate $(y)$ axis is vertical and directed downwards. Apart from a sample image itself, its binarised version as well as its vector skeleton were used for feature extraction.

Height, width, and height to width ratio were measured from the binarised image by determining the bounding box of the image. The bounding box coordinates $x_{1}, y_{1}, x_{2}, y_{2}$ correspond to the topmost, leftmost, bottommost, and rightmost black pixels on the image. The feature values were calculated as

$$
f_{1}=y_{2}-y_{1}+1, f_{2}=x_{2}-x_{1}+1, f_{3}=f_{1} / f_{2}
$$


Average stroke width $f_{12}$ was estimated from the number of foreground pixels $N_{s}$ and edge pixels $N_{D}$ in the binarised image as

$$
f_{12} \approx \frac{2 N_{s}}{N_{D}}
$$

Pseudo-pressure was estimated from the grey levels of the image pixels. Let the set of foreground pixels be $S$ and the intensity of a pixel be $I(x, y)$, black $=0 \leq$ $I(x, y) \leq 1=$ white. The average pseudo-pressure is thus

$$
f_{13}=\frac{1}{N_{S}} \sum_{\left(x_{i}, y_{i}\right) \in S} I\left(x_{i}, y_{i}\right)
$$

And the standard deviation of the pseudo-pressure is

$$
f_{14}=\sqrt{\frac{1}{N_{S}-1} \sum_{\left(x_{i}, y_{i}\right) \in S}\left(I\left(x_{i}, y_{i}\right)-f_{13}\right)^{2}}
$$

Other features were calculated from grapheme skeletons. The skeletons were produced by a specially developed content-dependent skeletonisation algorithm capable of producing skeletons that are very close to the human perception of the pen tip trajectory [21. The skeletonisation algorithm also restored some hidden loops. The resultant skeletons were presented as a set of spline-approximated strokes, including retraced strokes and loops.

Slant value was calculated by taking a set of sample points $s_{i}$ along each spline-approximated stroke that represented the element of interest (ascender, descender, etc.) and calculating the angles of tangents in these points $\alpha_{i}=$ $\arctan k_{i}$. The slants $f_{8}$ and $f_{9}$ were calculated as the weighted average of those angles:

$$
\alpha_{\text {slant }}=\frac{\sum_{i} l_{i} \alpha_{i}}{L}
$$

where $l_{i}$ is the length of the corresponding curve segment (see Figure 2(a)), $L=\sum_{i} l_{i}$ is the total curve length. The slant values were within the range $-\pi / 2 \leq \alpha_{\text {slant }}<\pi / 2$. To extract some features that represent relative position of characters ' $t$ ' and ' $h$ ' in the grapheme, the relative position of top stem points was measured along with the slants of the ' $h$ ' and ' $t$ ' stems. Detection of the top points was made by first detecting all the end points in the upper half of a sample image and then tracing the branches from those end points to determine which of them correspond to the elements of 'th'. All connected components in a sample image were detected. If the top of the stem points belonged to a different connected component, characters 't' and ' $h$ ' were disconnected, otherwise they were connected (binary feature $f_{11}$ ).

Number of strokes $f_{16}$ and number of loops and retraced strokes $f_{17}$ were available directly from the skeleton as well as the presence of loop at the top of t-stem and h-stem $\left(f_{21}\right.$ and $\left.f_{22}\right)$.

Standard deviation of stroke width $f_{15}$ was extracted by taking a set of closely sampled points on the skeleton curves and measuring the cross-section of the 
strokes on the underlying binarised image as shown in Figure 2(b) The obtained measurements were then used to calculate the feature value.

Straightness of a stroke (t-stem $f_{18}$, t-bar $f_{19}$, h-stem $f_{20}$ ) was calculated as follows: if the distance between the two end points was $d$ and the length of the stroke was $L$, the straightness of the stroke was given by

$$
\text { straightness }=\frac{L}{d}
$$

It was close to 1 for a straight stroke, and significantly larger for a curved stroke.

Maximum and average curvature of a stroke (h-knee) $f_{23}, f_{24}$ were calculated by taking closely sampled points along the curve, calculating the curvature at each point, and taking the largest value of the curvature and the average of the curvature.

Relative size of h-knee $f_{25}$ was calculated as the largest distance from h-stem to the h-knee curve as shown in Figure 2(c). It was approximately calculated as the largest horizontal distance between the curves representing the stem and the knee divided by the width of the grapheme $f_{2}$.

Algorithms for feature extraction consisted of a main program and subroutines for extraction of particular features. The input to the algorithms was a grapheme image, the output was the feature vector along with additional information which was later used to verify correctness of the feature values. Obviously it was impossible to write algorithms for all possible shapes of the grapheme. However, it was observed that for most samples of each grapheme a wide variety of shapes could be taken into account. Processing of input images was performed in several stages. In each stage some features were extracted and information about the grapheme form, such as position of certain points and correspondence of branches to certain parts of the grapheme, was refined. This information was then used in further stages of the feature extraction.

Sample images of grapheme 'th' for feature extraction were manually segmented from 600 images of the CEDAR letter [10] collected from 200 different writers. The samples were taken only from the beginning of words like 'the'. There were at most 27 samples of the grapheme extracted for each writer.

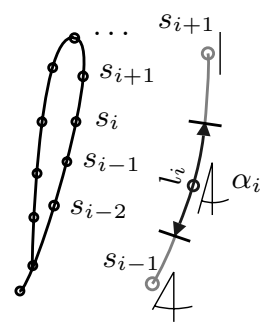

(a) Slant

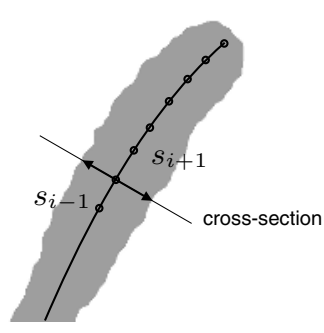

(b) Cross-section

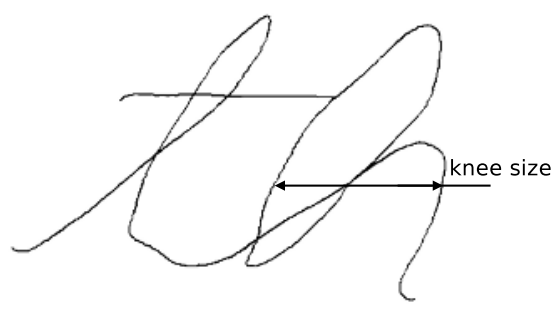

(c) H-knee size

Fig. 2. Some features of 'th' extracted from a vector skeleton 
Automatic feature extraction was carried out on the extracted samples of grapheme 'th' and the results were inspected visually. A sample was considered to be processed incorrectly if either some strokes were detected incorrectly, or the extraction algorithm failed to extract some features. The total extraction error rate was $6 \%$.

In order to make the results of the study comparable to those of the previous study when a raster skeletonisation method was used, samples from the same 165 different writers were chosen for writer accuracy evaluation experiments.

\section{Analysis of Feature Usefulness}

John et al. 22 analysed several definitions of feature relevance which have been presented in the literature and proposed a definition that includes two degrees of relevance: strong and weak relevance. Strong relevance means that a feature cannot be removed from the feature set without loss of classification accuracy. Weak relevance means that a feature can sometimes contribute to classification accuracy. A feature is irrelevant if it is neither strongly nor weakly relevant, and thus can be excluded from the feature set without loss of classification accuracy.

The definition of weak and strong feature relevance is hard to apply, since it is necessary to know the conditional probabilities $P\left(\vec{f}_{k} \mid j\right)$ of feature vector $\vec{f}_{k}$ represents a sample of $j$-th writer. Another approach can be applied. Suppose there is a set of features and all subsets are found that are equally good for writer classification. "Equally good" means that the classification accuracies achieved when those subsets are used, do not differ significantly - their average values are indistinguishable. There are three classes of features according to their inclusion in the found feature sets: some features are included in all feature sets, some are not included at all, and the rest are included in some of the sets. The first category of features comprises of indispensable features, the second category contains irrelevant features, and the rest of the features are partially relevant.

To find the best feature subsets, the wrapper approach was used. In this approach an induction algorithm is used for evaluation of a feature set. A feature set is assigned a value proportional to its performance - in this study this value was the accuracy of writer classification. The accuracy was evaluated using 5fold cross-validation [23. The training data was divided into 5 approximately equal parts and the induction algorithm was then run 5 times each time leaving one subset for test and using the other 4 parts for training. The classification accuracy obtained from the 5 tests was then averaged and associated with the corresponding feature subset.

DistAl, a constructive learning algorithm based on the multi-layer perceptron with spherical threshold units, was chosen as a classification system [24]. Use of a constructive artificial neural network has an advantage of not making any a priori assumption about the network topology. Instead, elements are added to the hidden layer as the learning progresses. The maximum number of 
elements can be restricted to avoid overfitting. The learning algorithm is fast because it does not use an iterative algorithm to compute perceptron parameters (weights, thresholds). The most time-consuming part is the calculation of inter-pattern distances for each pair of patterns. However, this needs to be performed only once for the whole data. Experiments conducted on both artificial and real data using the DistAl classifier [24] demonstrated classification results comparable to those obtained by other commonly used learning algorithms. Since, in the current study, it is not necessary to obtain the highest classification accuracy possible, but rather the focus is on comparing the classification accuracy of different feature subsets, the DistAl classifier suits the task well.

All feature values were treated as real numbers. Having performed several experiments we chose the normalised Manhattan distance as a distance measure for DistAl because this measure was shown to be suitable for the problem at hand:

$$
d\left(\vec{f}_{k_{1}}, \vec{f}_{k_{2}}\right)=\frac{1}{M} \sum_{i=1}^{M} \frac{\left|f_{i k_{1}}-f_{i k_{2}}\right|}{\max f_{i}-\min f_{i}}
$$

where $M$ was the number of features, and $\min f_{i}$ and $\max f_{i}$ were the minimum and maximum values of the $i$-th feature in the data set respectively.

A genetic algorithm (GA) was used to implement feature subset selection. From the studies of De Jong [25] GAs have been extensively used to solve problems of feature selection in pattern recognition [26-28]. Successful use of a GA together with the DistAl algorithm has also been demonstrated [29]. In this study GA with sharing [30] was used to find the best feature subsets. Original fitness $f i t_{s}$ for feature subset $x_{s}$ (represented as a binary string) was equal to the average correct classification rate $a c c_{s}$. Modified fitness was defined as

$$
\widetilde{f i t}_{s}=\frac{f i t_{s}}{\sum_{j}\left(1-d\left(x_{s}-x_{j}\right)\right)}
$$

where $d\left(x_{s}, x_{j}\right)$ is a dissimilarity measure:

$$
d\left(x_{s}, x_{j}\right)=\frac{\sum_{m}\left|x_{s m}-x_{j m}\right|}{M}
$$

Here $M$ is the number of features, or the length of binary strings $x_{s}$ and $x_{j}$, representing a feature subset, and $x_{s m}$ is the $m$-th bit of string $x_{s}$. Summation in eq. (8) is performed across the whole population of strings. The following parameters were used for the GA:

- population size of 50 ;

- uniform crossover 31] with probability of 0.6 ;

- mutation with probability of 0.03 ;

- replacement strategy in which best strings from parents and offspring form the next generation; 
- linear scaling of fitness function with the factor of 2 ;

- stop condition was "no new good feature subset during the last 50 generations".

The results of the experiments are presented below.

\section{Results and Conclusions}

There were a total of 3823 samples of grapheme 'th' taken from 165 different writers, with between 15 and 27 samples per writer. Accuracy of classification was measured by 5 -fold cross validation on the DistAl classifier. The best feature sets were searched using a genetic algorithm with sharing.

The optimal feature sets found are presented in Table 2] where $a$ is the average writer classification accuracy achieved when the associated feature set was used, and $\sigma_{a}$ is the standard deviation of the accuracy value. The feature values of writer classification accuracy presented in the table are indistinguishable at the $1 \%$ confidence level. In a string representing a feature set the value of $1(0)$ means that the feature is included in (excluded from) the set. The position of a digit in a string in Table 2 corresponds to the index of the feature. Table 1 should be consulted to get the name of the feature.

Based on the optimal feature subsets presented in Table 2, division of the features into three sets of indispensable, partially relevant, and irrelevant can be performed and is shown in Table 3. As seen from the table, there are only two irrelevant features in the set, and 17 out of total of 25 features are indispensable.

Features $f_{14}$ and $f_{15}$, the standard deviation of pseudo-pressure and standard deviation of stroke width were the two irrelevant features revealed by the experiment. The former feature was aimed at capturing the stability of pen pressure, measured from the gray level of an image. It is likely that evaluation of pen pressure from the image gray level is suitable to capture the overall darkness (pressure), but not suitable for capturing more subtle pressure variations. The latter feature was intended to partially represent a "line quality" feature [17. It seems that the variation of stroke width does not provide a useful information about individual line quality and rather represents a random noise which

Table 2. Optimal feature subsets of the 'th' feature set, accuracy values, and standard deviations. Bit 1 corresponds to presence of the feature in the subset, bit 0 to absence of it. Features are divided into groups of five for convenience.

\begin{tabular}{|c|c|}
\hline feature set $f_{1} \ldots f_{25}$ & $\sigma_{a}$ \\
\hline 1111111111110001110111111 & \begin{tabular}{|l|l|}
0.67 & 0.04 \\
\end{tabular} \\
\hline 1011111111110001110101111 & \begin{tabular}{|l|l|l}
0.67 & 0.04
\end{tabular} \\
\hline 1111111111111001110101111 & $0.65 \mid 0.05$ \\
\hline 1101111111111001111111111 & \begin{tabular}{|l|l|l|l}
0.64 & 0.04
\end{tabular} \\
\hline 1111111111111001110101111 & 40.04 \\
\hline 1111111111110001110100111 & 0.640 .04 \\
\hline
\end{tabular}


Table 3. Division of features according to their relevance. Feature indices are presented.

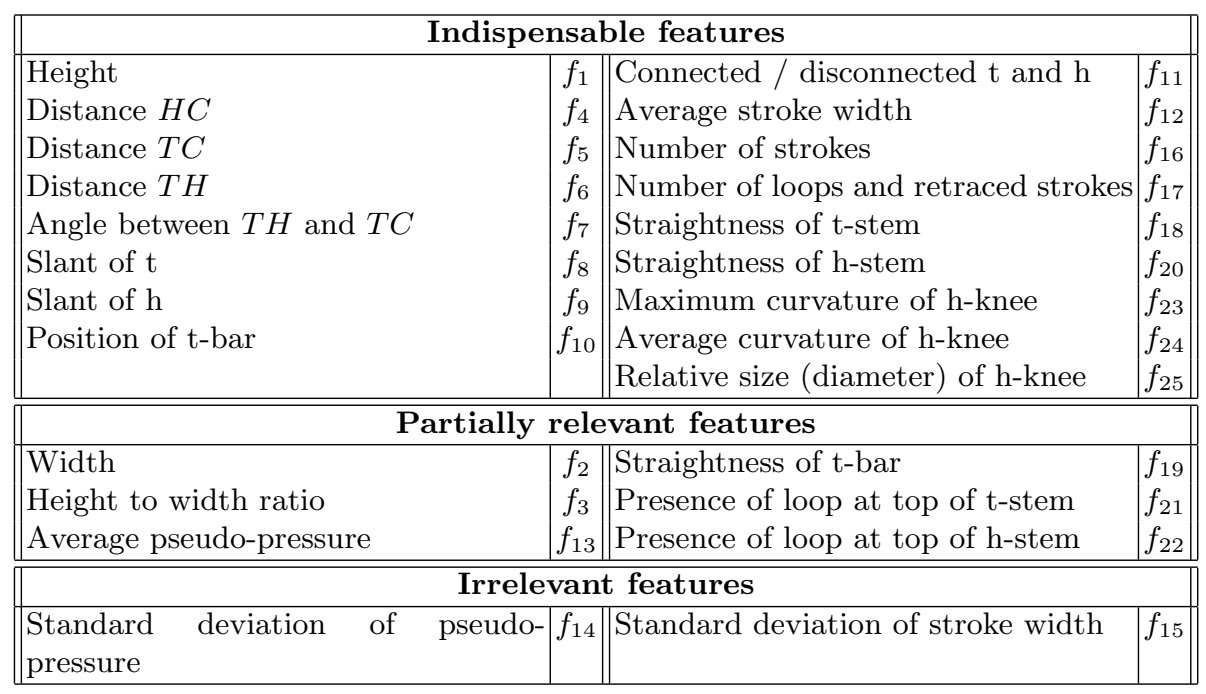

probably depends on writing conditions (pen, paper, writing surface), which were almost identical for all writers in the current study [10].

The errors in feature extraction were mostly due to wide variation of character shapes, some of which were not taken into account by feature extraction algorithms. For practical use of feature extraction algorithms in forensic document analysis tools an interactive extraction algorithms can be implemented on the base of the existing ones, which can improve the feature extraction accuracy further.

From the results obtained we conclude that a number of the features commonly used by forensic document examiners do possess discriminative power. Thus, use of those features for distinguishing writers by samples of their normal unconstrained handwriting is justified. How stable these features are under attempts at forgery or disguise needs to be studied further. Compared to the results obtained in our previous experiments, where the achieved writer classification accuracy on the same data set was $58 \%$ 20, use of the new skeletonisation algorithm allowed us to both improve feature extraction accuracy to $94 \%$ and extract more structural features, most of which contributed to the set of indispensable features, which in turn resulted in higher accuracy of writer classification of $67 \%$.

\section{References}

1. Hilton, O.: Scientific Examination of Questioned Documents. CRC Hall, Florida, USA (1993)

2. Robertson, E.W.: Fundamentals of Document Examination. Nelson-Hall (1991)

3. Harrison, W.R.: Suspect Documents, Their Scientific Examinations. Nelson-Hall, Illinois, USA (1981) 
4. Lindblom, B.: Document examination. In Chayko, G.M., Gulliver, E.D., eds.: Forensic Evidence in Canada. 2nd edn. Aurora: Canada Law Book (1999) 505-525

5. Daubert et al. v. Merrell Dow Pharmaceuticals. Court case 509 U. S. 579 (1993)

6. Kam, M., Fielding, G., Conn, R.: Writer identification by professional document examiners. Journal of Forensic Sciences 42 (1997) 778-786

7. Found, B., Sita, J., Rogers, D.: The development of a program for characterizing forensic handwriting examiners' expertise: Signature examination pilot study. Journal of Forensic Document Examination 12 (1999) 69-80

8. Kam, M., Gummadidala, K., Fielding, G., Conn, R.: Signature authentication by forensic document examiners. Journal of Forensic Science 46 (2001) 884-888

9. Sita, J., Found, B., Rogers, D.: Forensic handwriting examiners' expertise for signature comparison. Journal of Forensic Sciences 47 (2001) 1117-1124

10. Srihari, S.N., Cha, S.H., Arora, H., Lee, S.: Individuality of handwriting. Journal of Forensic Sciences 47 (2002) 1-17

11. Found, B., Roger, D., Schmittat, R.: A computer program designed to compare the spatial elements of handwriting. Forensic Science International 68 (1994) 195-203

12. Schomaker, L., Bulacu, M., van Erp, M.: Sparse-parametric writer identification using heterogeneous feature groups. In: Proc. 3rd Int'l Conf. Document Analysis and Recognition (ICDAR'95), Montreal, Canada (1995) 545-548

13. Srihari, S.N., Cha, S.H., Lee, S.: Establishing handwriting individuality using pattern recognition techniques. In: Proc. 6th Int'l Conf. Document Analysis and Recognition (ICDAR'2001), Seattle, USA (2001) 1195-1204

14. Tomai, C.I., Zhang, B., Srihari, S.N.: Discriminatory power of handwritten words for writer recognition. In: Proc. 17th Int'l Conf. Pattern Recognition, Cambridge, UK (2004) 638-641

15. Zhang, B., Srihari, S.N., Lee, S.: Individuality of handwritten characters. In: Proc. 7th Int'l Conf. Document Analysis and Recognition (ICDAR'2003), Edinburgh, UK (2003) 1086-1090

16. Srihari, S.N., Tomai, C.I., Zhang, B., Lee, S.: Individuality of numerals. In: Proc. 7th Int'l Conf. Document Analysis and Recognition (ICDAR'2003), Edinburgh, UK (2003) 1096-1100

17. Huber, R.A., Headrick, A.M.: Handwriting Identification: Facts and Fundamentals. CRC Press, LCC (1999)

18. Leedham, C.G., Pervouchine, V., Tan, W.K., Jacob, A.: Automatic quantitative letter-level extraction of features used by document examiners. In Teulings, H.L., Van Gemmert, A.W.A., eds.: Proc. 11th Conf. Int'l. Graphonomics Society (IGS2003), Scottsdale, AZ, USA (2003) 291-294

19. Leedham, C.G., Pervouchine, V., Tan, W.K.: Quantitative letter-level extraction and analysis of features used by document examiners. Journal of Forensic Document Examination (2004) In press.

20. Leedham, C.G., Pervouchine, V.: Validating the use of handwriting as a biometric and its forensic analysis. In Pal, U., Parui, S.K., Chaudhuri, B.B., eds.: Document Analysis: Proc. Int'l. Workshop on Document Analysis (IWDA'05). Allied Publishers Ltd., Chennai (2005) 175-192 Invited lecture.

21. Pervouchine, V., Leedham, C.G., Melikhov, K.: Handwritten character skeletonisation for forensic document analysis. In: Proc. 20th Annual ACM Symposium on Applied Computing, Santa Fe, NM, USA (2005) 754-758

22. John, G.H., Kohavi, R., Pfleger, K.: Irrelevant features and the subset selection problem. In: Proc. 11th Int'l Conf. Machine Learning (ML94), Rutgers University, New Brunswick, NJ (1994) 121-129 
23. Weiss, S.M., Kulikowski, C.A.: Computer Systems that Learn: Classification and Prediction Methods from Statistics, Neural Nets, Machine Learning, and Expert Systems. Morgan Kaufmann (1991)

24. Yang, J., Parekh, R., Honavar, V.: Distal: An inter-pattern distance-based constructive learning algorithm. Technical Report ISU-CS-TR 97-06, Department of Computer Science, Iowa State University (1997) Also appeared in Proc. Int'l. Conf. Neural Networks, IEEE, Piscataway, N.J, 1998.

25. Vafaie, H., De Jong, K.: Genetic algorithms as a tool for feature selection in machine learning. In: Proc. 4th Int'l Conf. Tools with Artificial Intelligence (TAI'92), Arlington, VA, IEEE Computer Society Press (1992) 200-203

26. Brill, F.Z., Brown, D.E., Martin, W.N.: Fast genetic selection of features for neural network classifiers. IEEE Trans. Neural Networks 3 (1992) 324-328

27. Bala, J., Huang, J., Vafaie, H., De Jong, K., Wechsler, H.: Hybrid learning using genetic algorithms and decision trees for pattern classification. In: Proc. Int'l Joint Conf. Artificial Intelligence (IJCAI-95), Montreal, Canada (1995)

28. Chen, S., Smith, S., Guerra-Salcedo, C., Whitley, D.: Fast and accurate feature selection using hybrid genetic strategies. In: Proc. Congress on Evolutionary Computation (CEC99), Washington DC, USA (1999)

29. Yang, J., Honavar, V.: Feature subset selection using a genetic algorithm. IEEE Intell. Syst. 13 (1998) 44-49

30. Goldberg, D.E.: Genetic Algorithms in Search, Optimization, and Machine Learning. Addison-Wesley (1989)

31. Syswerda, G.: Uniform crossover in genetic algorithms. In: Proc. 3rd Int'l Conf. Genetic Algorithms, George Mason University, USA, Morgan Kaufmann (1989) 2-9 